\title{
The Electronic Structure of Nonpolyhex Carbon Nanotubes ${ }^{\dagger}$
}

\author{
István László* \\ Department of Theoretical Physics, Institute of Physics and Center for Applied Mathematics, \\ Budapest University of Technology and Economics, H-1521 Budapest, Hungary
}

Received August 25, 2003

\begin{abstract}
Generalizing the folding method to any periodic two-dimensional planar carbon structures we have calculated the corresponding electronic structures in the framework of the one orbital one site tight-binding (BlochHückel) method by solving the eigenvalue problems in a numerical way. We discussed the metallic or the nonmetallic behavior of the nanotubes by applying the folding vectors of parameters $(m, n)$. We extended the topological coordinate method to two-dimensional periodic planar structures as well. Nearly regular hexagonal, pentagonal, and heptagonal polygons were obtained. The curvatures of the final relaxed structures can be read from the sizes of the polygons. Thus relying only on the topological information we could describe the shape of the tubular structures and their conductivity behaviors.
\end{abstract}

\section{INTRODUCTION}

All polyhex single wall nanotubes can be indexed by the $(m, n)$ pair of integers, and in the one orbital one site tightbinding calculations the nanotube electronic structures can be constructed from that of the graphene sheet. ${ }^{1,2}$ These $m, n$ integers are associated with a lattice vector $\mathbf{s}_{\mathbf{1}}=m \mathbf{a}_{\mathbf{1}}+n \mathbf{a}_{\mathbf{2}}$, along which the graphene sheet is rolling up. Here $\mathbf{a}_{1}$ and $\mathbf{a}_{2}$ are the unit vectors of the primitive unit cell containing two atoms. If $n-m$ is a multiple of 3 , then the tube is metallic, and in other cases it is nonmetallic. There are also analytical relations for the energy levels. ${ }^{1,2}$ The case of the nonpolyhex nanotubes is much more complicated. ${ }^{3-17}$ There is not yet an available general formula for the geometric and electronic structures of the nonpolyhex nanotubes when they contain pentagons and heptagons as well. ${ }^{18,19}$

In ref 20 we presented a method for describing the geometrical structures of nonhexagonal nanotubes, nanocoils, and nanotori. Starting from the topological arrangement of carbon atoms and using the topological coordinates we constructed good initial Cartesian coordinates for nanotubes, and their final structures were obtained with the help of a Brenner-potential $^{21}$ based molecular mechanics calculation. For a given tiling the toroidal, helical, or tubular relaxed structure depends on the position and shape of the super cell to be rolled up.

The $\mathbf{R}^{2}$ plane cannot be always tilled by regular hexagons, pentagons, and heptagons. Here we present a topological coordinate method for the tiling of the two-dimensional plane by near regular polygons. The size of the polygons will be proportional to the Gaussian curvature of the relaxed structure. Small sizes of polygons correspond to positive Gaussian curvatures, and great sizes of polygons correspond to negative ones. The Gaussian curvature is the product of the two principal curvatures.

In the one orbital one site tight-binding (with the other name of Hückel-type) calculations the electronic structure

\footnotetext{
Dedicated to Dr. George W. A. Milne, a long-term Editor-in-Chief of JCICS.

* Corresponding author e-mail: laszlo@phy.bme.hu.
}

of the graphene sheet can be used in the description of the nanotube electronic structures by applying the folding method. In this paper we shall extend this method to any two-dimensional periodic tiling of the plane and to the corresponding tubular, helical, and toroidal structures. In the reciprocal $\mathbf{k}$ space the electronic eigenvalue problem will be solved in a numerical way.

\section{THE TOPOLOGICAL COORDINATES}

There are already developed topological coordinates for fullerenes, ${ }^{22,23}$ tori, ${ }^{24,25}$ and nanotubes. ${ }^{20}$ Here we extend them to periodic planar structures as well. Using this method we can do preliminary calculations for several carbon structures without applying any relaxation process. Let us suppose that we know only the number of carbon atoms and the connectivity structure. That is for each atom we know the neighboring atoms. Using this information the adjacency matrix $\mathbf{A}$ can be constructed with matrix elements $A_{i j}=1$ if $i$ and $j$ are adjacent and $A_{i j}=0$ otherwise. In an appropriate energy unit of this paper $\mathbf{A}$ is equal to the minus of the Hückel Hamiltonian $\mathbf{H}$. The topological coordinates are constructed from the so-called bilobal eigenvectors ${ }^{22,23}$ of the adjacency matrix.

Vectors having this bilobal property can be identified in the following way. For a candidate vector color black all vertices bearing positive coefficients, white all bearing negative coefficients, and gray all bearing a zero coefficient; now delete all gray vertices, all edges incident on gray vertices, and all edges connecting a black to a white vertex; if the graph now consists of exactly two connected components, one of black and one of white vertices then the eigenvector is of the bilobal type. ${ }^{22,23,25}$

\section{THE CASE OF TWO-DIMENSIONAL PERIODIC PLANAR STRUCTURES}

The topological coordinates for a two-dimensional periodic planar structure can be constructed in a way very similar to 
a

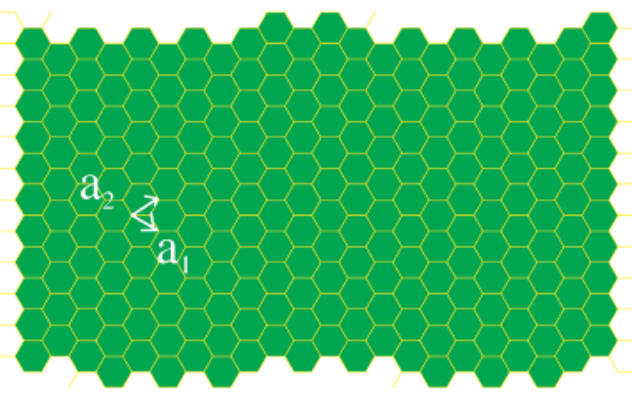

C

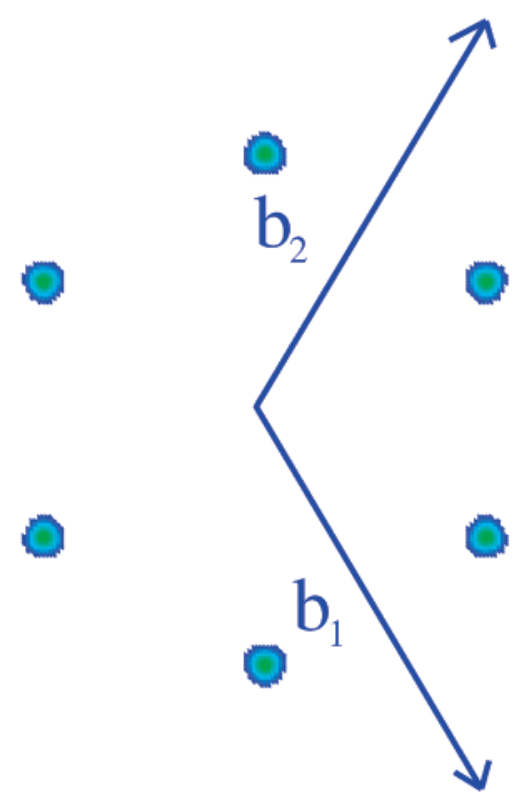

0.3 b

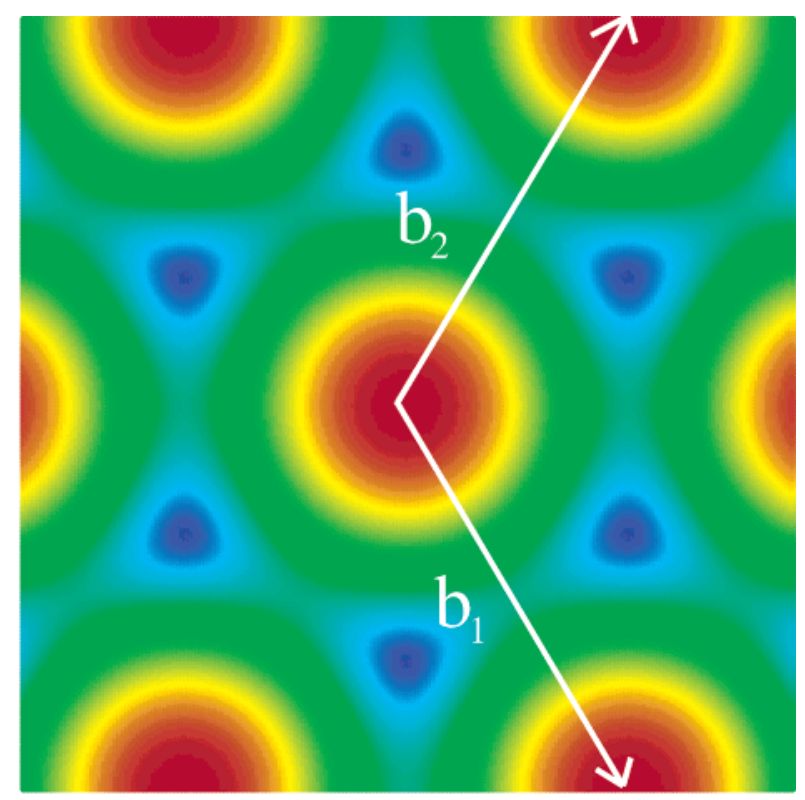

0.0

d
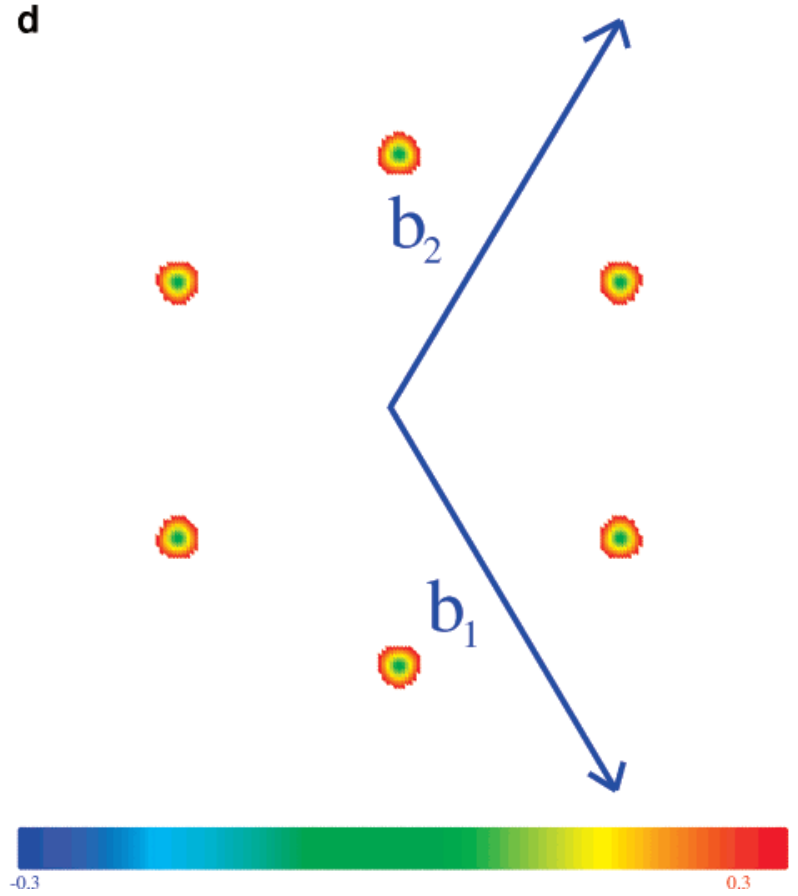

Figure 1. The hexagonal planar structure. a. The topological coordinates. b. The $E_{\mathrm{HOMOk}}-E_{\mathrm{LumOk}}$ energy gap in the function of $\mathbf{k}$ and in the range of energy $(0.0,6.0)$. c. The $E_{\text {HOMOk }}$ energy in the function of $\mathbf{k}$ and in the range of energy $(-0.3,0.3)$. d. The $E_{\mathrm{LUMOk}}$ energy in the function of $\mathbf{k}$ and in the range of energy $(-0.3,0.3)$.

the case of the tubular structures. ${ }^{20}$ First we construct a super cell or parallelogram in the periodic two-dimensional planar structure, then by identifying the opposite edges of the parallelogram we obtain an auxiliary torus. In this identification the directions of the opposite edges are the same. The topological coordinates of this torus are constructed in the way presented for the torus, and a simple geometric transformation gives the original parallelogram. Thus the topological coordinates of a two-dimensional periodic planar structure are the following:

$$
x_{\mathrm{i}}=r \arccos \left(S_{4} C_{i}^{k_{4}} / r\right) \text { if } C_{i}^{k_{3}} \geq 0
$$

and

$$
\begin{gathered}
x_{i}=-r \arccos \left(S_{4} C_{i}^{k_{4}} / r\right) \text { if } C_{i}^{k_{3}}<0 \\
y_{i}=0 \\
z_{i}=R \arccos \left(S_{1} C_{i}^{k_{1}} / R\right) \text { if } C_{i}^{k_{2}} \geq 0
\end{gathered}
$$

and

$$
z_{i}=R\left(2 \pi-\arccos \left(S_{1} C_{i}^{k_{1}} / R\right)\right) \text { if } C_{i}^{k_{2}}<0
$$


a

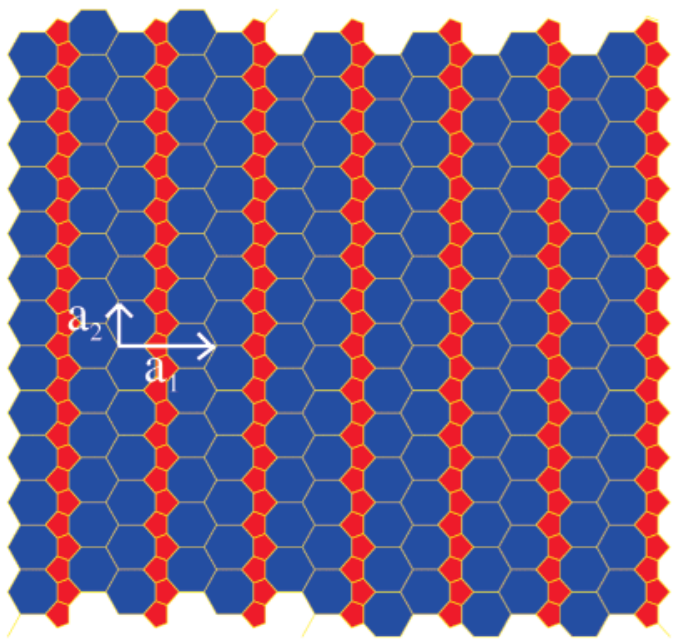

b

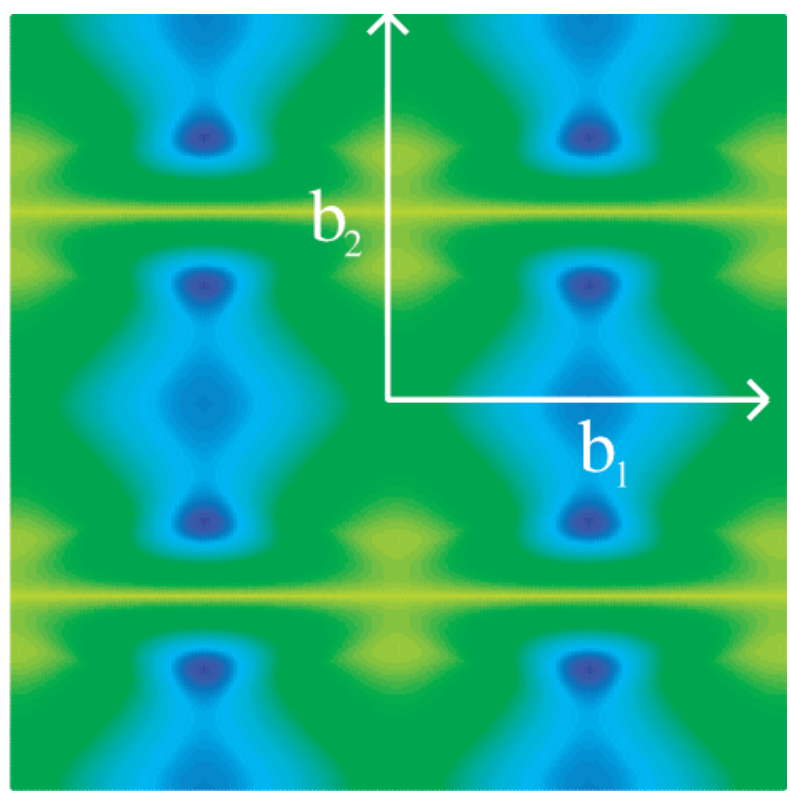

0.0

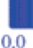

d

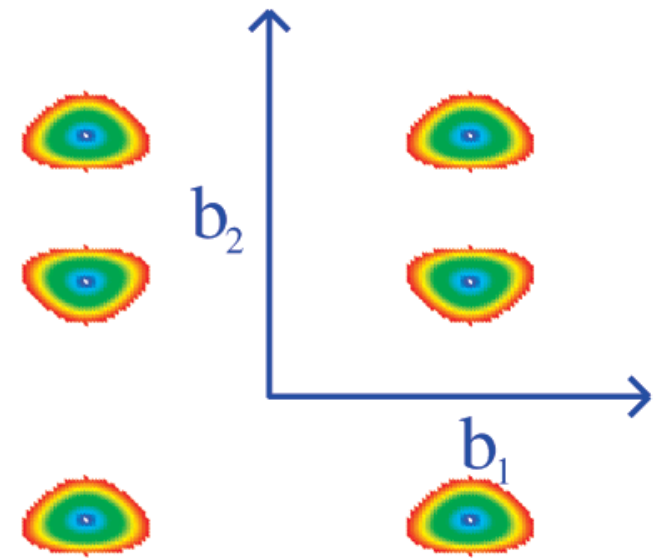

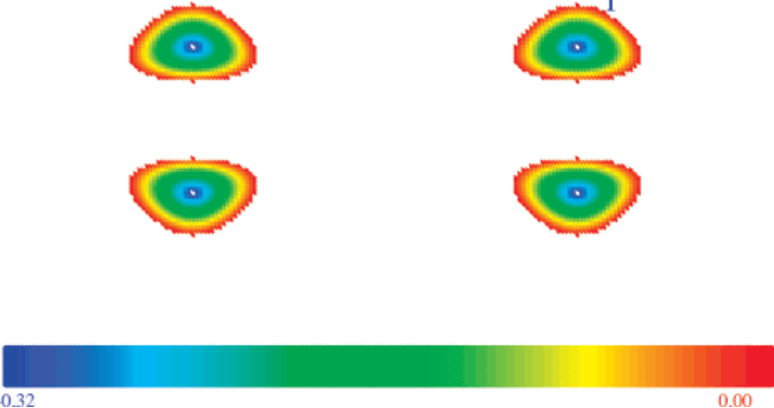
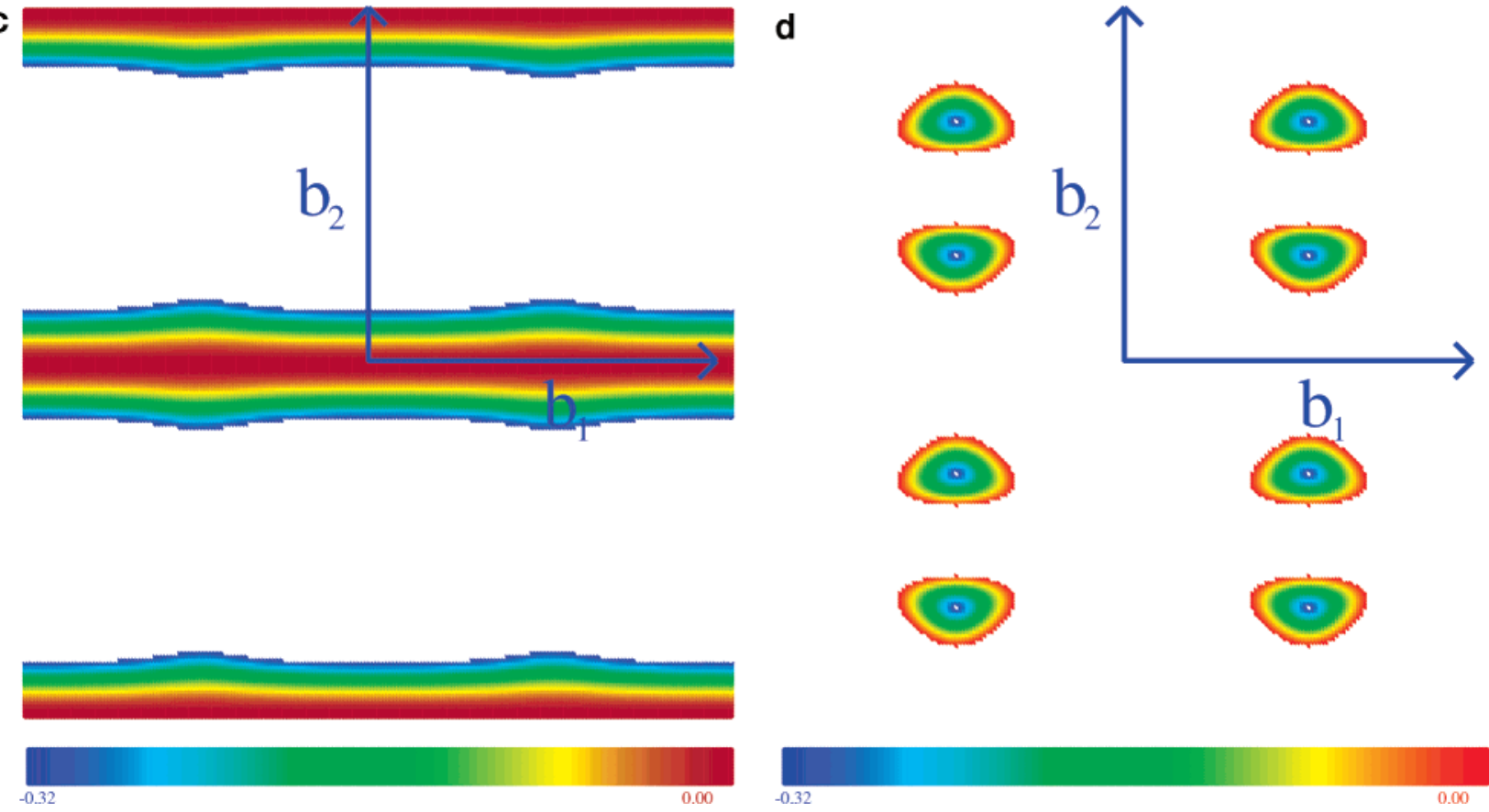

Figure 2. The planar structure of Figure 1 in ref 20. a. The topological coordinates. b. The $E_{\mathrm{HOMOk}}-E_{\mathrm{LumOk}}$ energy gap in the function of $\mathbf{k}$ and in the range of energy $(0.0,2.1)$. c. The $E_{\mathrm{HOMO}}$ energy in the function of $\mathbf{k}$ and in the range of energy $(-0.32,0.0)$. d. The $E_{\mathrm{LuMO}}$ energy in the function of $\mathbf{k}$ and in the range of energy $(-0.32,0.0)$.

Here $\boldsymbol{c}^{k_{1}}, \boldsymbol{c}^{k_{2}}, \boldsymbol{c}^{k_{3}}$, and $\boldsymbol{c}^{k_{4}}$ are the four bilobal eigenvectors of the adjacency matrix $\mathbf{A}$ of the auxiliary torus. The radii $R$ and $r$ govern the size and aspect ratio of the torus. At each point $\left(x_{\mathrm{i}}, y_{\mathrm{i}}, z_{\mathrm{i}}\right)=\mathbf{R}_{i}+\mathbf{r}_{i}$ we used the additional scaling for $R=\left|\mathbf{R}_{i}\right|$ and $r=\left|\mathbf{r}_{i}\right|$ (see ref 20).

As an example let us see the case of a two-dimensional hexagonal structure. It can be generated by the translations, $\mathbf{t}=n_{1} \mathbf{a}_{\mathbf{1}}+n_{2} \mathbf{a}_{\mathbf{2}}$, where $n_{1}$ and $n_{2}$ are integers and $\mathbf{a}_{1}$ and $\mathbf{a}_{2}$ are unit vectors of the direct lattice. Each unit cell contains two carbon atoms which are atom numbers 1 and 2. The neighboring structure of the lattice is totally described by giving the neighbors for each atom in the $\left(n_{1}, n_{2}\right)=(0,0)$ unit cell. That is atom 1 of unit cell $(0,0)$ has the number 2 atom as the neighbor in the unit cells $(0,0),(-1,0)$, and $(0$, $-1)$, and atom 2 of unit cell $(0,0)$ has the number 1 neighboring atoms in the unit cells $(0,0),(0,1)$, and $(1,0)$. If for example the unit vectors of the super cell are $\mathbf{s}_{\mathbf{1}}=$ $12 \mathbf{a}_{1}+12 \mathbf{a}_{2}$ and $\mathbf{s}_{2}=-12 \mathbf{a}_{1}+12 \mathbf{a}_{2}$ the adjacency matrix A of the auxiliary torus is obtained by identifying the opposite edges and using the before mentioned neighboring structure. In this special case the $\mathbf{c}^{2}, \mathbf{c}^{3}, \mathbf{c}^{4}$, and $\mathbf{c}^{5}$ are the four bilobal eigenvectors and eqs $1-5$ give the structure of Figure 1a with $\mathbf{c}^{k_{1}}=\mathbf{c},{ }^{4} \mathbf{c}^{k_{2}}=\mathbf{c},{ }^{5} \mathbf{c}^{k_{3}}=\mathbf{c}^{2}$, and $\mathbf{c}^{k_{4}}=\mathbf{c}^{3}$. The structures in Figures $2 \mathrm{a}-5 \mathrm{a}$ are constructed in the same way. They contain pentagons, hexagons, and heptagons, and their topological structures are described in ref 20 . 
a

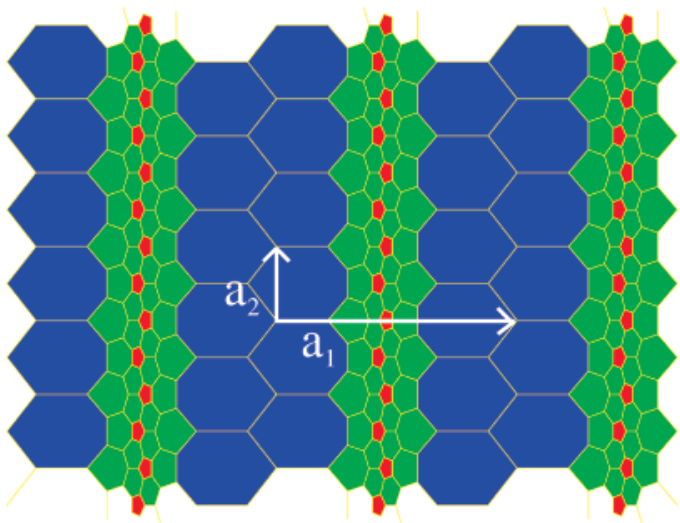

c
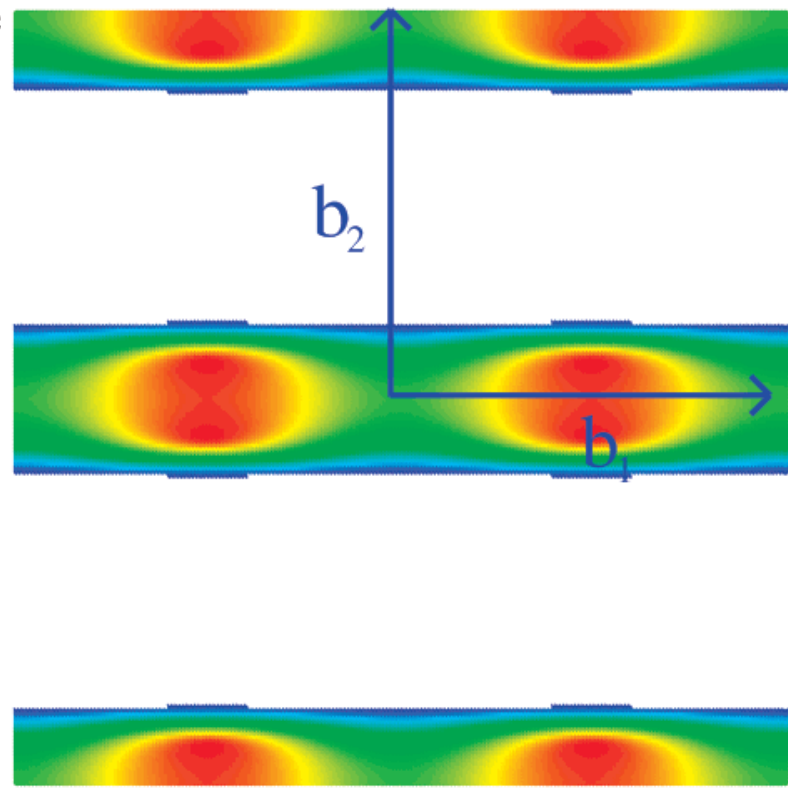

$-0.12$

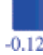

0.11 b

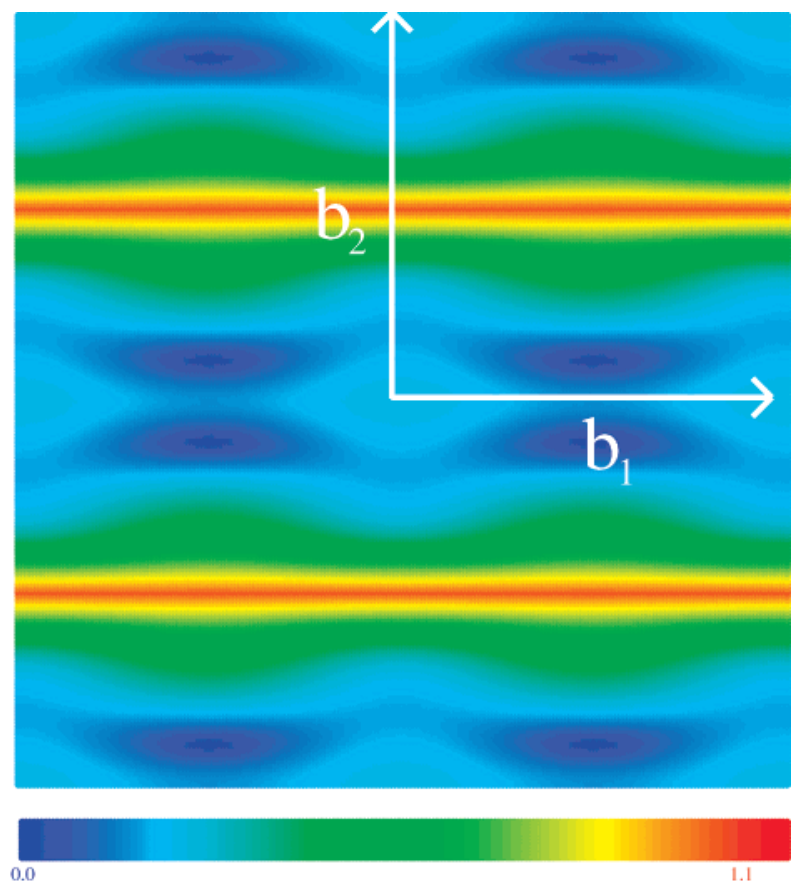

d
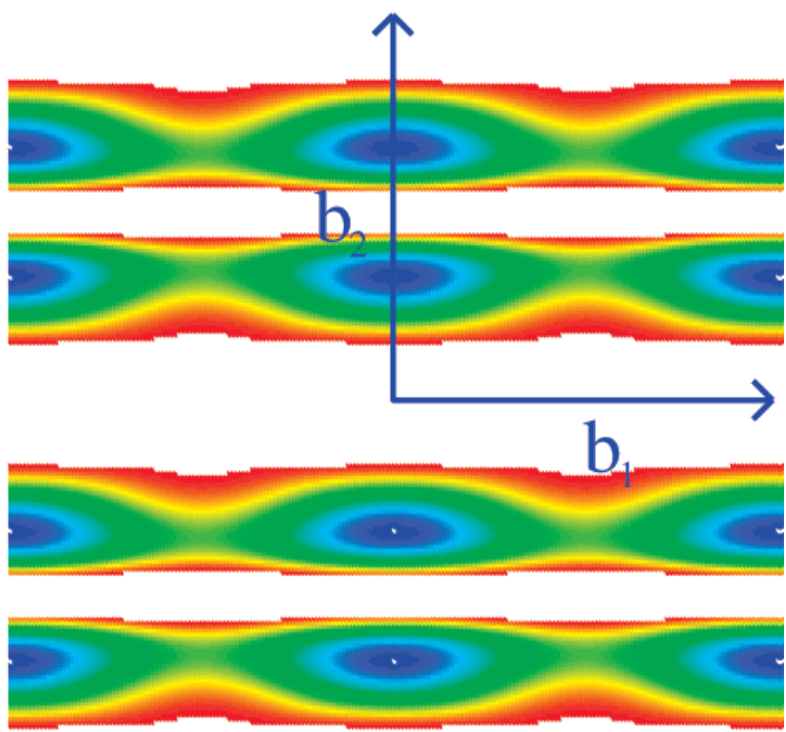

$-0.12$

Figure 3. The planar structure of Figure 2 in ref 20. a. The topological coordinates. b. The $E_{\mathrm{HOMOk}}-E_{\mathrm{LUMOk}}$ energy gap in the function of $\mathbf{k}$ and in the range of energy $(0.0,1.1)$. c. The $E_{\mathrm{HOMO}}$ energy in the function of $\mathbf{k}$ and in the range of energy $(-0.12,0.11)$. d. The $E_{\mathrm{LUMOk}}$ energy in the function of $\mathbf{k}$ and in the range of energy $(-0.12,0.11)$.

\section{THE ELECTRONIC STRUCTURE OF NANOTUBES}

The simplest method of studying the electronic structure of nanotubes is the one orbital one site tight-binding method or in the terminology of quantum chemistry the Bloch-Hückel approximation. As in this approximation the nanotube electronic structure can be constructed from that of the infinite sheet, ${ }^{1,2}$ first we study the two-dimensional periodic planar structure.

From the $\mathbf{a}_{1}$ and $\mathbf{a}_{2}$ unit cell vectors of the direct lattice the $\mathbf{b}_{1}$ and $\mathbf{b}_{\mathbf{2}}$ unit cell vectors of the reciprocal lattice are calculated by the relations

$$
\begin{aligned}
& \mathbf{b}_{1}=2 \pi \frac{\mathbf{a}_{2} \times \mathbf{z}}{\left(\mathbf{a}_{1} \cdot \mathbf{a}_{2} \times \mathbf{z}\right)} \\
& \mathbf{b}_{2}=2 \pi \frac{\mathbf{z} \times \mathbf{a}_{1}}{\left(\mathbf{a}_{1} \cdot \mathbf{a}_{2} \times \mathbf{z}\right)}
\end{aligned}
$$

where $\mathbf{z}$ has the same direction as $\mathbf{a}_{\mathbf{1}} \times \mathbf{a}_{\mathbf{2}}$ with $\mathbf{z} \cdot \mathbf{z}=1$. The wave functions of wavenumber $\mathbf{k}=k_{1} \mathbf{b}_{1}+k_{2} \mathbf{b}_{2}$ are

$$
|\mu \mathbf{k}\rangle=\sum_{\nu=1}^{r} c_{\mu \mathbf{k}}^{v}|\mathbf{k}\rangle_{v}
$$

with 
a

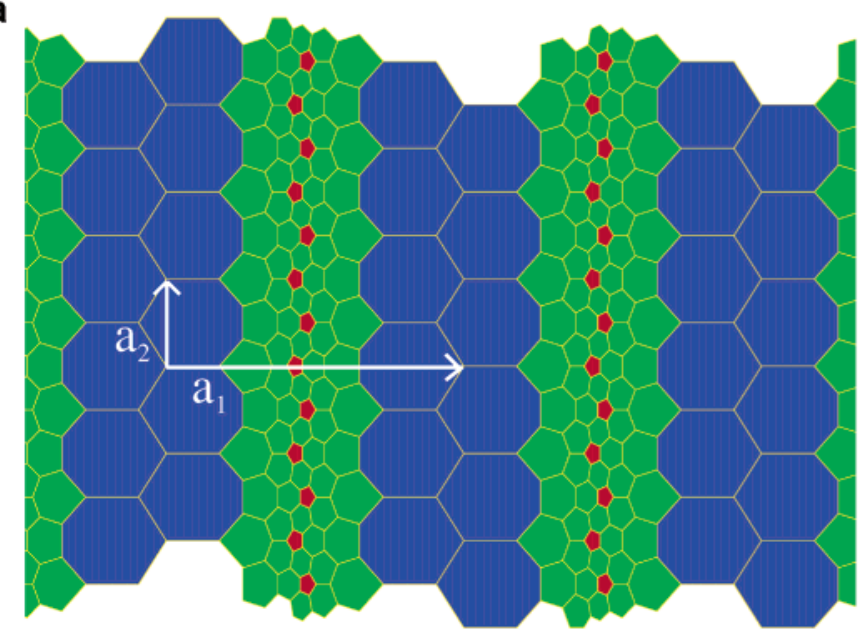

C
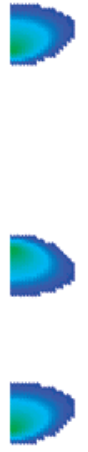

$b_{2}$
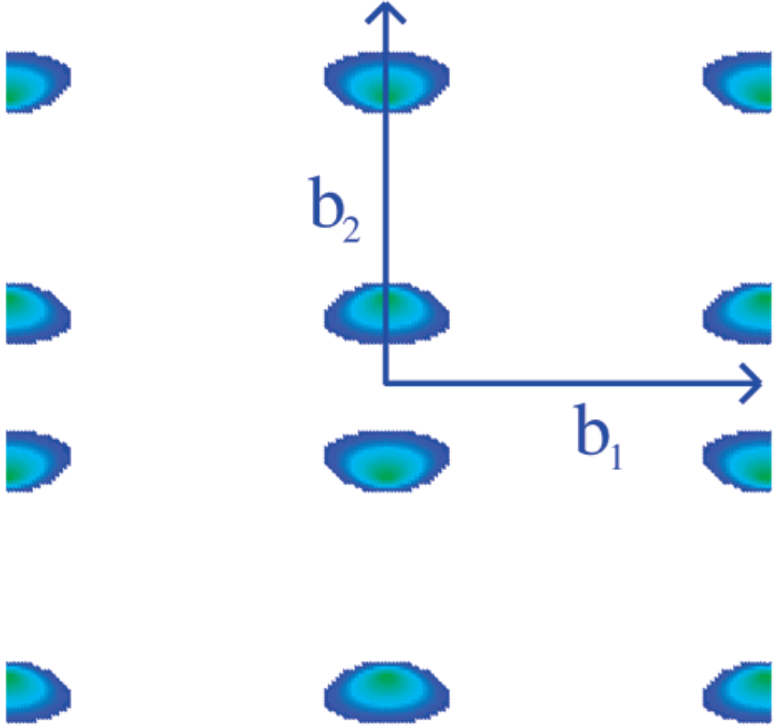

$-0.2$

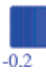

Figure 4. The planar structure of Figure 3 in ref 20. a. The topological coordinates. b. The $E_{\mathrm{HOMOk}}-E_{\mathrm{LumOk}}$ energy gap in the function of $\mathbf{k}$ and in the range of energy $(0.0,1.0)$. c. The $E_{\mathrm{HOMO}}$ energy in the function of $\mathbf{k}$ and in the range of energy $(-0.2,0.0)$. d. The $E_{\mathrm{LuMO}}$ energy in the function of $\mathbf{k}$ and in the range of energy $(-0.2,0.0)$.

$$
|\mathbf{k}\rangle_{v}=\frac{1}{\sqrt{N}} \sum^{\mathbf{t}} e^{i \mathbf{k} t}|\mathbf{t}\rangle_{v}
$$

where $r$ is the number of atoms in the unit cell, $\mu=1,2, \ldots$, $r$ is the band index, $\mathbf{t}=n_{1} \mathbf{a}_{1}+n_{2} \mathbf{a}_{2}$ is the translations of the lattice, and $|\mathbf{t}\rangle_{v}$ is the atomic basis function centered at the site $v$ of the unit cell $\mathbf{t}$. $n_{1}$ and $n_{2}$ are integers, $k_{1}$ and $k_{2}$ are real numbers, and $v=1,2, \ldots, r$. The number of unit cells is $N .{ }^{2,26}$ The $c_{\mu \mathbf{k}}^{v}$ coefficients are the $v$ th eigenvectors of the $H(\mathbf{k})$ Hamiltonian and $E_{v \mathbf{k}}$ is the corresponding eigenvalue, where

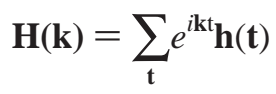

b
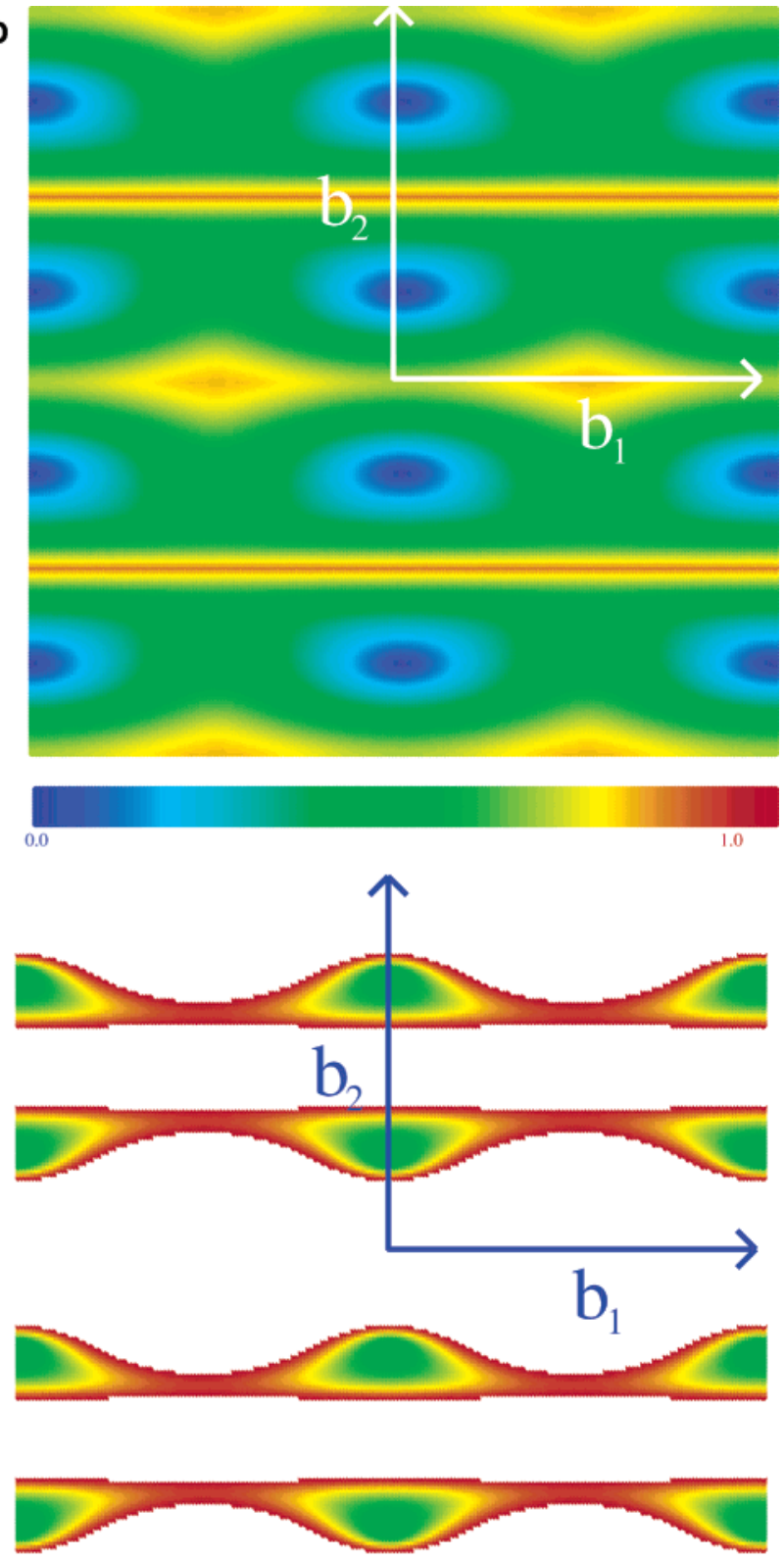

and

$$
h(\mathbf{t})_{\mu \nu}=\left\langle\mathbf{0}^{\mu}\left|h_{\text {eff }}\right| \mathbf{t}^{\nu}\right\rangle
$$

Here $\left\langle 0^{\mu}\left|h_{\text {eff }}\right| \mathbf{t}^{\nu}\right\rangle=-1$ if the $\mu$ th atom of the 0 unit cell and the $v$ th atom of the $\mathbf{t}$ unit cell are neighbors and $\left\langle 0^{\mu}\left|h_{\mathrm{eff}}\right| \mathbf{t}^{\nu}\right\rangle$ $=0$ in other cases. ${ }^{26}$ If the neighbors of an atom are no farther than the neighboring unit cell of the atom in question, the summation for $\mathbf{t}$ [in eq 10] means the summation for the unit cells $(0,0),(1,0),(-1,0),(0,1),(0,-1),(1,1),(-1$, $-1),(1,-1)$, and $(-1,1)$.

In this Bloch-Hückel-type tight-binding calculation the electronic structure of the planar sheet can be used for the electronic structure calculation of the single-walled nano- 
a

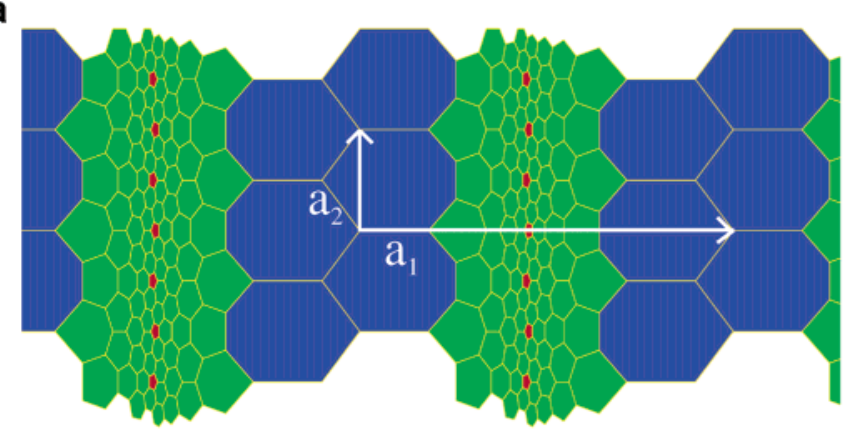

C
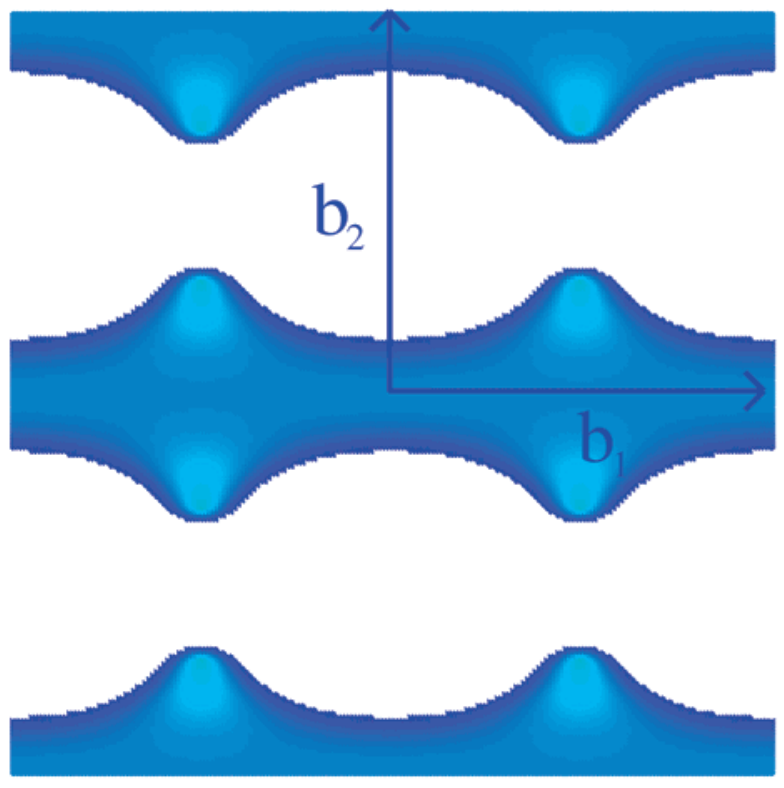

$-0.2$ b
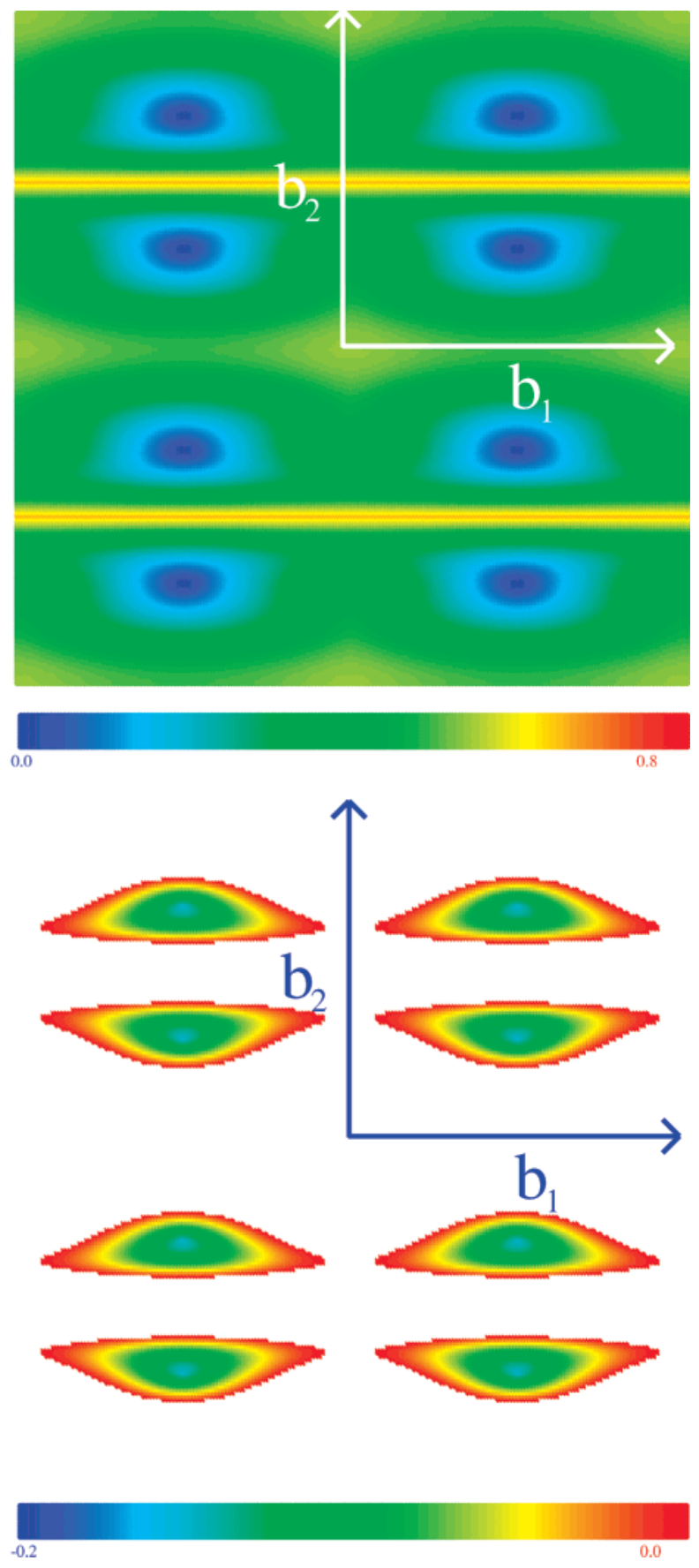

Figure 5. The planar structure of Figure 4 in ref 20. a. The topological coordinates. b. The $E_{\mathrm{HOMOk}}-E_{\mathrm{LumOk}}$ energy gap in the function of $\mathbf{k}$ and in the range of energy $(0.0,0.8)$. c. The $E_{\mathrm{HOMOk}}$ energy in the function of $\mathbf{k}$ and in the range of energy $(-0.2,0.0)$. d. The $E_{\mathrm{LuMOk}}$ energy in the function of $\mathbf{k}$ and in the range of energy $(-0.2,0.0)$.

tubes. One part of an infinite long nanotube can be obtained from the super cell of the side vectors $\mathbf{s}_{1}=m \mathbf{a}_{1}+n \mathbf{a}_{2}, \mathbf{s}_{\mathbf{2}}=$ $p \mathbf{a}_{1}+q \mathbf{a}_{2}$ by identifying the opposite sides parallel with $\mathbf{s}_{2}$. ( $m, n, p$, and $q$ are integers.) The periodic boundary condition on the vector $\mathbf{s}_{\mathbf{1}}$ is fulfilled if

$$
\mathbf{s}_{\mathbf{1}} \mathbf{k}=2 \pi l_{1}
$$

where $l_{1}$ is an integer. Thus the allowed $\mathbf{k}$ values are on parallel lines in the reciprocal space. As $\mathbf{k}=k_{1} \mathbf{b}_{1}+k_{2} \mathbf{b}_{2}$ and $\mathbf{s}_{\mathbf{1}}=m \mathbf{a}_{\mathbf{1}}+n \mathbf{a}_{2}$, eq 12 can be simplified by eqs 6 and 7 as

$$
m k_{1}+n k_{2}=l_{1}
$$

where $k_{1}$ and $k_{2}$ are real values. The $\mathbf{k}$ values in the first reciprocal lattice unit cell can be characterized by $\left(0 \leq k_{1}\right.$ $<1)$ and $\left(0 \leq k_{2}<1\right)$. From eq 13 it follows that if we are restricted to the first reciprocal unit cell, then the number of possible $l_{1}$ values is $n+m$ for $n$ and $m$ having the same sign, and $|n|+|m|-1$ if they have different signs.

If both sides of the super cell are identified we obtain the toroidal structures and must apply periodic boundary conditions for the vector $\mathbf{S}_{\mathbf{2}}$ as well. With this condition we obtain the following restrictions for the allowed $\mathbf{k}$ values

$$
\mathbf{s}_{\mathbf{2}} \mathbf{k}=2 \pi l_{2}
$$

and 


$$
p k_{1}+q k_{2}=l_{2}
$$

with the integer $l_{2}$ values. The two periodic boundary conditions give that only isolated $\mathbf{k}$ values are allowed for toroidal structures.

\section{RESULTS}

The drawings obtained from the topological coordinates of various planar atomic arrangements are presented in Figures 1a, 2a, 3a, 4a, and 5a. For the polyhex structure we obtained a regular hexagonal atomic arrangement (Figure 1a). In the other structures however the sizes of the heptagons are great and those of the pentagons are small. The size of hexagons decreases by increasing the heptagon-hexagon distances and it increases by increasing the pentagonhexagon distances. From these topological coordinates we can read information about the shape of the relaxed nanotubes constructed by the folding method of the super cell. Let us construct for example a tube from a given super cell having great heptagons and small pentagons on its surface. The Gaussian curvature of the tube is zero, namely one of the principal curvatures is zero in this case. To obtain the realistic carbon-carbon distances, the local distances around the pentagons must be increased and around the heptagons must be decreased. This can be reached by indentures around the heptagons and by bulging around the pentagons. Thus the small sizes in the drawings by the topological coordinates correspond to the positive Gaussian curvatures and the great sizes to the negative ones. The zero curvature is attributed to the average carbon-carbon distances.

Now we turn to the study of the electronic structures. For each energy level $E_{v \mathbf{k}}$ we defined the $E_{v \min }=\min \left(E_{v \mathbf{k}}\right)$ and $E_{v \max }=\max \left(E_{v \mathbf{k}}\right)$ minimum and maximum values in the function of $\mathbf{k}$. As a supposed occupation of the orbitals, we define for each $\mathbf{k}$ the Highest Occupied Molecular Orbital $E_{\mathrm{HOMOk}}$ and the Lowest Unoccupied Molecular Orbital $E_{\mathrm{LUMOk}}$, supposing that for each $\mathbf{k}$ the lowest $r / 2$ levels are occupied and the other highest levels are unoccupied. These are not however always the highest occupied or lowest unoccupied molecular orbitals in the ground state. From the definition it follows that $E_{\mathrm{LUMOk}}-E_{\mathrm{HOMOk}} \geq 0$, and in our cases the $r$ number of $\pi$-electrons is even. If $E_{\text {HOMOmax }}<$ $E_{\mathrm{LUMOmin}}$ then $E_{\mathrm{HOMOk}}$ is really the Highest Occupied Molecular Orbital and $E_{\text {LUMOk }}$ is really the Lowest Unoccupied Molecular Orbital in the ground state of the system. This is not the case if $E_{\text {HOMOmax }}>E_{\mathrm{LUMOmin}}$ when the ground-state occupation numbers do not correspond to the before mentioned supposed occupations. Now the Fermi-level is over $E_{\text {LUMOmin }}$ and under $E_{\text {HOMOmax }}$.

In Figure $1 \mathrm{~b}$ we can see the $E_{\mathrm{LUMOk}}-E_{\mathrm{HOMOk}}$ function for the polyhex structure, and Figure 1c,d shows that the $E_{\mathrm{HOMOk}}=E_{1 \mathrm{k}}$ and $E_{\mathrm{LUMOk}}=E_{2 \mathrm{k}}$ bands coincide only in special points, the $K$ points.

There are only $r=2$ atoms in the unit cell and $E_{\text {HOMOmax }}$ $=E_{\text {LUMOmin }}=0$. Taking advantage of the hexagonal symmetry it can be proved that only the nanotubes obeying the condition $m-n=0(\bmod 3)$ are metallic., ${ }^{1,2}$ That is only in these cases the lines of eqs 12 and 13 are crossing the $K$ points.

In Figures $2-5$ the reciprocal lattice unit vectors are scaled in such a way that $\left|\mathbf{b}_{\mathbf{1}}\right|=\left|\mathbf{b}_{\mathbf{2}}\right|$ and the eigenvalue problem of the Hamiltonian $\mathbf{H}(\mathbf{k})$ is solved in a numerical way. The $r$ number of atoms in the unit cell are 8, 16, 24, 32, and 60 in order for the structures in Figures 2a, 3a, 4a, and 5a. For the structures in Figures 2 and $3 E_{\text {HOMOmax }}>E_{\text {LUMOmin }}$ and for the structures in Figures 4 and $5 E_{\text {HOMOmax }} \approx E_{\text {LUMOmin }}$. In Figures $2 \mathrm{c}, 2 \mathrm{~d}, 3 \mathrm{c}$, and $3 \mathrm{~d}$ the energy levels are presented only in the range ( $\left.E_{\mathrm{LUMOmin}}, E_{\mathrm{HOMOmax}}\right)$, and the white color corresponds to values out of this range. Let us see a nanotube with the parameters $(m, n)=(1,0)$. From eq 13 it follows that the allowed $k$ values in the first reciprocal unit cell are characterized with $k_{1}=0$. Figure $2 \mathrm{c}, \mathrm{d}$ shows that for this line the supposed lowest unoccupied energy levels are always higher than the supposed highest occupied energy levels. The nanotube will not be metallic. The same figures show that the nanotube $(m, n)=(3,-5)$ will be metallic, as one of the allowed $\mathbf{k}$ line will be $3 k_{1}=5 k_{2}$. This line crosses such a region in Figure 2c where the energy levels are occupied, and it crosses such a region in Figure $2 \mathrm{~d}$ where the unoccupied levels have lower energy values than some occupied ones in Figure 2c. Thus this supposed occupation of energy levels is not a ground state, and some electrons most go to such energy levels which were not supposedly occupied in the drawing of the figures. This is why this tube becomes metallic. We shall have metallic tubes for $(m, n)$ $=(1,-1)$ and nonmetallic for $(m, n)=(0,5)$. From Figure $2 b-d$ it follows that in nonmetallic nanotubes the smallest electronic transitions correspond to indirect energy gaps. Similar analysis of Figure $3 b-d$ shows that the nanotubes $(1,0),(1,-1),(3,-5)$, and $(0,5)$ are metallic and the nanotubes $(0,1)$ and $(0,2)$ are nonmetallic.

As in Figures 4 and 5 we have the relation $E_{\text {HомOmax }} \approx$ $E_{\text {LUMOmin }}$. Here the situation is very similar to the case of the polyhex structure, but there is not a closed formula for the conditions of conductivity. We must do the same analysis as for the previous structures. In Figure 4 we obtained that the nanotubes $(1,-1),(3,-5),(0,5),(0,1)$, and $(0,2)$ are nonmetallic, and the nanotube $(1,0)$ is metallic. In Figure 5 the nanotubes $(1,0),(1,-1),(0,5),(0,2)$, and $(0,1)$ are nonmetallic and the nanotube $(3,-5)$ is metallic.

\section{CONCLUSION}

Starting from the topological arrangements of carbon atoms we discussed the electronic structures of the nonpolyhex carbon nanotubes. The calculations are based on the Bloch-Hückel theory (or the one orbital one site tight-binding method). More sophisticated methods can change some results, but the main tendencies can be found in these cases as well such as in the polyhex carbon structures. We have found that the extension of the topological coordinate method can be used in the drawing of various two-dimensional periodic carbon arrangements, and it is useful also in the estimating of the final Gaussian curvatures in the relaxed structures. Thus relying only on the topological information we described the shape of the tubular structures and their conductivity behaviors.

\section{ACKNOWLEDGMENT}

This work was supported by grants from PAST and OTKA (T 038191, T043231). The author thanks Prof. André Rassat ${ }^{27}$ for the fruitful discussions. 


\section{REFERENCES AND NOTES}

(1) Dresselhaus, M. S.; Dresselhaus, G.; Eklund, P. C. Science of Fullerenes and Carbon Nanotubes; Academic Press: San Diego, Boston, New York, London, Sydney, Tokyo, Toronto, 1996; Chapter 19.

(2) Ceulemans, A.; Chibotaru, L. F.; Bovin, S. A.; Fowler, P. W. The Electronic Structure of Polyhex Carbon Tori. J. Chem. Phys. 2000, 112, 4271-4278.

(3) Mackay, A. L.; Terrones, H. Diamond from graphite. Nature 1991, 352,762 .

(4) Ihara, S.; Itoh, S.; Kitakami, J.-i. Toroidal Forms of Graphitic Carbon. Phys. Rev. B 1993, 47, 12908-12911.

(5) Ihara, S.; Itoh, S.; Kitakami, J.-i. Helically Coiled Cage Forms of Graphitic Carbon. Phys. Rev. B 1993, 48, 5643-5647.

(6) Itoh, S.; Ihara, S. Toroidal Forms of Graphitic Carbon. II. Elongated Tori. Phys. Rev. B 1993, 48, 8323-8328.

(7) Kirby, E. C. On Toroidal Azulenoids and Other Shapes of Fullerene Cage. Fullerene Sci. Technol. 1994, 2, 395-404.

(8) Amelinckx, S.; Zhang, X. B.; Bernaerts, D.; Zhang, X. F.; Ivanov, V.; Nagy, J. B. A Formation Mechanism for Catalytically Grown Helix-shaped Graphite Nanotubes. Science 1994, 265, 635-639.

(9) Avron, J. E.; Berger, J. Tiling Rules for Toroidal Molecules. Phys. Rev. A 1995, 51, 1146-1149.

(10) Avron, J. E.; Berger, J. Classification Scheme for Toroidal Molecules. J. Chem. Soc., Faraday Trans. 1995, 91, 4037-4045.

(11) László, I.; Rassat, A. Toroidal and Spherical Fullerene-like Molecules with Only Pentagonal and Heptagonal Faces. Int. J. Quantum Chem. 2001, 84, 136-139.

(12) Diudea, M. V. Toroidal Graphenes from 4-valent Tori. Bull. Chem. Soc. Japan 2002, 75, 487-492.

(13) Diudea, M. V. Topology of Naphthylenic Tori. Phys. Chem. Chem. Phys. 2002, 4, 4740-4746.

(14) Diudea, M. V.; John, P. E.; Graovac, A.; Primorac, M.; Pisanski, T. Leapfrog and Related Operations on Toroidal Fullerenes. Croat. Chem. Acta 2003, 76, 153-159.

(15) Bíró, P. L.; Márk, G. I.; Koós, A. A.; B.Nagy, J.; Lambin, Ph. Coiled Carbon Nanotube Structures with Supraunitary Nonhexagonal Ring
Ratio. Phys. Rev. B 2002, 66, 165405-1-165405-6.

(16) Xia, J. Y.; Hosoya, H. Analysis of the Relationship among the Graphs Isomorphic to Multilayered Cyclic Fence Graphs (MLCFG). J. Chem Inf. Comput. Sci. 2002, 42, 1004-1010.

(17) Réti, T.; Böröczky, K. Topological Characterization of 2D Finite Cellular Systems. Mater. Sci. Forum 2003, 414, 471-481.

(18) Akagi, K.; Tamura, R.; Tsukada, M.; Itoh, S.; Ihara, S. Electronic Structure of Helically Coiled Cage of Graphitic Carbon. Phys. Rev Lett. 1995, 74, 2307-2310.

(19) Akagi, K.; Tamura, R.; Tsukada, M.; Itoh, S.; Ihara, S. Electronic Structure of Helically Coiled Carbon Nanotubes: Relation Between the Phason Lines and Energy Band Features. Phys. Rev. B 1996, 53 , 2114-2120.

(20) László, I.; Rassat, A. The geometric structure of deformed nanotubes and the topological coordinates. J. Chem. Inf. Comput. Sci. 2003, 43, 519-524.

(21) Brenner, D. W. Empirical Potentials for Hydrocarbons for Use in Simulating the Chemical Vapor Deposition of Diamond Films. Phys. Rev. B 1990, 42, 9458-9471.

(22) Manolopoulos, D. E.; Fowler, P. W. Molecular Graphs, Point Groups, and Fullerenes. J. Chem. Phys. 1992, 96, 7603-7614.

(23) Fowler, P. W.; Manolopoulos D. E. An Atlas of Fullerenes; Clarendon Press: Oxford, 1995; Chapter 5, pp 101-104.

(24) Graovac, A.; Plavšić, D.; Kaufman, M.; Pisanski, T.; Kirby, E. C Application of the Adjacency Matrix Eigenvectors Method to Geometry Determination of Toroidal Carbon Molecules. J. Chem. Phys. 2000, 113, 1925-1931.

(25) László, I.; Rassat, A.; Fowler, P. W.; Graovac, A. Topological Coordinates for Toroidal Structures. Chem. Phys. Lett. 2001, 342, 369-374.

(26) Del Re, G.; Ladik, J.; Biczó, G. Self-Consistent-Field Tight-Binding Treatment of Polymers. I. Infinite Three-Dimensional Case. Phys. Rev 1967, 155, 997-1003.

(27) Rassat, A. Département de Chimie, Ecole Normale Supérieure, 24, rue Lhomond, F-75231 Paris Cedex 05, France.

CI030413U 